\title{
AN EXPLICIT FORMULA FOR THE EULER NUMBERS OF HIGHER ORDER
}

\author{
QIU-MING LUO
}

Abstract. The object of the present note is to prove a new explicit formulae for the Euler numbers of higher order.

In the usual notations, let $E_{n}^{(\alpha)}$ denote the Euler numbers of highter order, defined by (see $[1$, p.66, Eq.(65)])

$$
\left(\frac{2 e^{z}}{e^{2 z}+1}\right)^{\alpha}=\sum_{n=0}^{\infty} E_{n}^{(\alpha)} \frac{z^{n}}{n !}, \quad\left(|z|<\frac{\pi}{2}\right)
$$

for an arbitrary (real or complex) parameter $\alpha$.

Recently, Q.-M. Luo, Y.-M. Zheng, and F. Qi [2, p.2, Eqs.(9) and (10)] gave two new classes of recursion formulae for the Euler numbers of higher order. In the present note we first prove the following explicit formula for the Euler numbers of higher order:

$$
E_{n}^{(\alpha)}=\sum_{k=0}^{n} \frac{(-1)^{k}}{2^{k}}\left(\begin{array}{c}
\alpha+n \\
n-k
\end{array}\right)\left(\begin{array}{c}
\alpha+k-1 \\
k
\end{array}\right) \sum_{j=0}^{k}\left(\begin{array}{l}
k \\
j
\end{array}\right)(k-2 j)^{n}
$$

Proof. By the generating relation (1) yields

$$
E_{n}^{(\alpha)}=\left.D_{z}^{n}\left\{\left(\frac{2}{e^{z}+e^{-z}}\right)^{\alpha}\right\}\right|_{z=0}, \quad D_{z}=\frac{d}{d z} .
$$

Since

$$
(1+w)^{-\alpha}=\sum_{l=0}^{\infty}\left(\begin{array}{c}
\alpha+l-1 \\
l
\end{array}\right)(-w)^{l}, \quad(|w|<1),
$$

setting $1+w=\left(e^{z}+e^{-z}\right) / 2$, and applying the binomial theorem, we find form (3) that

$$
E_{n}^{(\alpha)}=\left.\sum_{l=0}^{n}\left(\begin{array}{c}
\alpha+l-1 \\
l
\end{array}\right) \sum_{k=0}^{l} \frac{(-1)^{k}}{2^{k}}\left(\begin{array}{l}
l \\
k
\end{array}\right) D_{z}^{n}\left\{\left(e^{z}+e^{-z}\right)^{k}\right\}\right|_{z=0}
$$

Received and revised March 23, 2004.

2000 Mathematics Subject Classification. Primary 11B68, 33E20.

Key words and phrases. Euler numbers, Euler numbers of higher order. 
again applying the binomial theorem readily yields

$$
\left.D_{z}^{n}\left\{\left(e^{z}+e^{-z}\right)^{k}\right\}\right|_{z=0}=\sum_{j=0}^{k}\left(\begin{array}{c}
k \\
j
\end{array}\right)(k-2 j)^{n}
$$

and upon substituting this value in (4), yields

$$
E_{n}^{(\alpha)}=\sum_{l=0}^{n}\left(\begin{array}{c}
\alpha+l-1 \\
l
\end{array}\right) \sum_{k=0}^{l} \frac{(-1)^{k}}{2^{k}}\left(\begin{array}{l}
l \\
k
\end{array}\right) \sum_{j=0}^{k}\left(\begin{array}{l}
k \\
j
\end{array}\right)(k-2 j)^{n}
$$

if we rearrange the resulting triple series (5), we have

$$
\begin{aligned}
E_{n}^{(\alpha)} & =\sum_{k=0}^{n} \frac{(-1)^{k}}{2^{k}} \sum_{j=0}^{k}\left(\begin{array}{c}
k \\
j
\end{array}\right)(k-2 j)^{n} \sum_{l=0}^{n-k}\left(\begin{array}{c}
\alpha+k+l-1 \\
k+l
\end{array}\right)\left(\begin{array}{c}
k+l \\
k
\end{array}\right) \\
& =\sum_{k=0}^{n} \frac{(-1)^{k}}{2^{k}}\left(\begin{array}{c}
\alpha+k-1 \\
k
\end{array}\right) \sum_{j=0}^{k}\left(\begin{array}{c}
k \\
j
\end{array}\right)(k-2 j)^{n} \sum_{l=0}^{n-k}\left(\begin{array}{c}
\alpha+k+l-1 \\
l
\end{array}\right) .
\end{aligned}
$$

The innermost sum in (6) can be evaluated by appealing to the elementary combinatorial identity:

$$
\sum_{j=0}^{s}\left(\begin{array}{c}
\lambda+l-1 \\
l
\end{array}\right)=\left(\begin{array}{c}
\lambda+s \\
s
\end{array}\right)
$$

and leads us immediately to the explicit formula (2).

Finally, in view of the special case of our formula (2) when $\alpha=1$ provide an interesting result for the classial Euler numbers $E_{n} \equiv E_{n}^{(1)}$ :

$$
E_{n}=\sum_{k=0}^{n} \frac{(-1)^{k}}{2^{k}}\left(\begin{array}{l}
n+1 \\
k+1
\end{array}\right) \sum_{j=0}^{k}\left(\begin{array}{l}
k \\
j
\end{array}\right)(k-2 j)^{n} .
$$

\section{Acknowledgements}

The author was supported in part by NNSF(\#10001016) of China, SF for the Prominent Youth of Henan Province (\#0112000200).

\section{References}

[1] H. M.Srivastava and J. Choi, Series Associated with the Zeta and Related Functions, Kluwer Academic Publishers, Dordrecht, Boston, and London, 2001.

[2] Q.-M. Luo, Y.-M. Zheng and F. Qi, Euler numbers of higher order and Euler polynomials of higher order, henan Sci. 21(2003), 1-6. (In China). 
[3] H. M. Srivastava, Some formulae for the Bernoulli and Euler polynomials at eational arguments. Math. Proc. Cambridge Philos. Soc. 129(2000), 77-84.

[4] Srivastava H. M. and Todorov P. G., An explicit formula for the generalized Bernoulli polynoaials, J. Math. Anal. Appl. 130 (1988), 509-513.

Department of Mathematics, Jiaozuo University, Jiaozuo City, Henan 454003, The People's Republic of China.

E-mail: luomath@126.com 